Sociologie et sociétés

SOCIOLOGIE

ET SOCIÉTÉS

\title{
Jeunes et espaces urbains. Bilan des recherches en France,
} 1977-1994

\section{The Young and Urban Spaces. Review of Research in France,} 1977-1994

\section{Michel KOKOREFF}

Volume 28, numéro 1, printemps 1996

Les jeunes

URI : https://id.erudit.org/iderudit/001389ar

DOI : https://doi.org/10.7202/001389ar

Aller au sommaire du numéro

\section{Éditeur(s)}

Les Presses de l'Université de Montréal

\section{ISSN}

0038-030X (imprimé)

1492-1375 (numérique)

Découvrir la revue

Citer cet article

KOKOREFF, M. (1996). Jeunes et espaces urbains. Bilan des recherches en France, 1977-1994. Sociologie et sociétés, 28(1), 159-176.

https://doi.org/10.7202/001389ar

\section{Résumé de l'article}

Dans un contexte marqué par la crise urbaine, la fin du plein emploi et la remise en cause des institutions de socialisation, on assiste depuis quelques années en France à un regain d'intérêt pour la question du rapport entre les jeunes et la ville. Proposer un bilan provisoire de ces travaux, en repérer les lignes de force et de transformation, mais aussi les obstacles et limites, constitue l'objet du présent article. On fait l'hypothèse que ces travaux attestent des formes différenciées de l'inscription sociale des jeunes dans les espaces urbains. 


\section{Jeunes et espaces urbains. Bilan des recherches en France, 1977-1994}

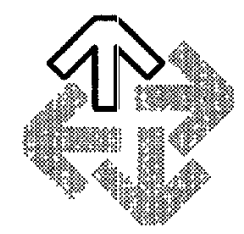

MICHEL KOKOREFF

La prise en compte de la dimension spatiale des conduites des jeunes présente-t-elle un apport spécifique à la sociologie de cette classe d'âge et des processus sociaux qui la définissent ? Faut-il y voir, à l'inverse, le produit d'une conjoncture et de modes qui se traduisent par une « demande sociale » forte, et réduisent la portée heuristique d'une telle approche?

Certes, la question n'est pas « nouvelle » : elle sous-tend toute une tradition de recherche sur l'assise territoriale des phénomènes de délinquance juvénile et des formations sociales correspondantes, et elle traverse les débats suscités par les recherches urbaines. Cependant, elle se trouve réactualisée, tout particulièrement dans le cas de la France contemporaine - retenu ici pour son exemplarité - par divers phénomènes sociaux.

Dans un contexte marqué par la crise urbaine, la fin du plein emploi et la remise en cause des institutions de socialisation, on assiste depuis quelques années à un regain d'intérêt pour la question du rapport entre les jeunes et la ville. En témoigne un faisceau d'indicateurs : le volume des publications ${ }^{1}$, la multiplication des colloques, journées d'études, séminaires ${ }^{2}$, la constitution de revues de synthèse ${ }^{3}$. Une diversification des centres d'intérêt a vu le jour autour de la problématique jeunes/identité/espaces urbains, qu'il s'agisse d'explorer les nouvelles figures cle la déviance juvénile et les incivilités qu'elles impliquent, de rendre compte des articulations entre espace et socialisation, précarité ou exclusion et logiques territoriales, ou de saisir

1. Afin de limiter le corpus étudié, on a pris comme critère la publication, délaissant les rapports de recherche, les mémoires et thèses. Pour les ouvrages, se distinguent deux catégories : la première contient explicitement le rapport « jeunes »/ wville ", voir notamment Augustin, 1991 ; Roulleau-Berger, 1991 ; Lesourd (éd.), 1992 ; Barreyre et . Vulbeau (éd.), 1994 ; la seconde aborde la situation sociale dans les banlieues françaises tout en faisant de la population-jeuries un élément d'analyse central ; voir Dubet, 1987 ; Calogirou, 1989 ; Dubet, Lapeyronnie, 1992 ; Jazouli, 1992 ; Duprez, Heddli, 1992 ; Roman (éd.), 1994 ; Vieillard-Baron, 1994. Pour les revues, si cette distinction est à l'ouvre, elle est compliquée par la coexistence de numéros spéciaux et d'articles isolés, mais aussi compte tenu de la question de leur légitimité académique variable. En ce qui concerne les thèmes traités ici, on dira que les revues les plus significatives se situent entre un pôle académique et un pôle professionnel (cf. Les Annales de la recherche urbaine, Les Cahiers de la sécurité intérieure, Esprit).

2. On peut citer, parmi des colloques plus généralistes, Les tags et la ville, CFE-PJJ, Vaucresson, 1990 ; L'actualité des bandes, CRIV, 1991 ; La jeunesse et la ville, Société française de sociologie, 1992 ; Du stade au quartier, IDEF/ INJEP, 1993 ; Jeunesses et politiques d'insertion, Commissariat Général du Plan, 1994.

3. Voir par exemple, Banlieue,ville et lien social, (Paris VIII) ; Bachmann in G. Férreol (1992); Blöss in La ville, Courrier du CNRS, n ${ }^{\circ} 81$, été 1994. 
comment l'espace est sollicité (ou pas) comme variable opérante dans le cadre des politiques publiques. Proposer un bilan provisoire de ces travaux, en repérer les lignes de force et de transformation, mais aussi les obstacles et limites, constitue l'objet du présent article.

Il convient auparavant de délimiter le champ et d'expliciter d'entrée les problèmes qu'il pose. Trois questions tendent en effet à s'y confondre, qui renvoient à des registres d'analyse différents et à une chronologie restant à établir. Il y a tout d'abord la question des «banlieues » - catégorie devenue générique - , réactualisée par l'irruption de violences urbaines et de la mobilisation collective des nouvelles générations issues de l'immigration, qui symbolise aujourd'hui l'accumulation et la localisation des « problèmes sociaux » dans les quartiers réputés « en difficultés ». Il y a ensuite la question de la «ville » considérée comme enjeu social majeur tout au long des années quatre-vingt, qui s'est traduite par le renouvellement des politiques urbaines dont la "politique de la ville " n'est qu'un aspect face à une recomposition d'ensemble des rapports sociaux. Il y a enfin la question dite des « jeunes dans la ville », incarnée notamment par l'accès au logement, aux équipements ou au centre-ville, la compatibilité/ incompatibilité de leurs pratiques avec l'espace local, qui est loin de se réduire aux jeunes marginalisés issus des classes populaires qui défrayent la chronique périodiquement. Leur distinction analytique est un préalable afin d'éviter les amalgames entre les catégories utilisées, les usages métonymique et métaphorique de l'expression « jeunes des banlieues », les causalités trop rapidement instituées, et par là, de participer à leur construction sociale.

Ensuite, les travaux cités sont porteurs d'un paradoxe : la dimension spatiale de « la jeunesse » est peu explorée en tant que telle. Les glissements sont constants entre « effets de milieu " (les adolescents dans la cité) et "situations " (les jeunes de ou à la rue), décor (la jeunesse $e n$ banlieue ) et métaphore (l'espace social des jeunes); et ce, au détriment du rapport entre une population donnée et des espaces (les étudiants et leur territoire). On retrouve là un problème général posé par le vocable d'« urbain » et la notion d'《espace ». Celle-ci est plus souvent mobilisée par les sociologues comme métaphore (Grafmeyer, 1994) qu'objectivée comme dimension participant au faisceau des déterminations économiques, culturelles, temporelles de la vie sociale (Rémy et Voye, 1992); elle est plus sollicitée par sociologisme comme question que dans sa valeur opératoire (Ostrowetsky, 1994). Délaissée par les sociologues, l'approche socio-spatiale a surtout été développée par des géographes renforçant la méfiance des premiers vis-à-vis d'un déterminisme «spatial». Il semble pourtant que cette approche soit aujourd'hui en plein renouvellement face aux nécessités sur le plan théorique et pratique de penser les tensions entre le global et le local, le macro et le micro, les échelles de territorialisation du «nous ».

Cela étant posé, l'objectif de cet article est de mettre en relief la constitution progressive de ce domaine de recherche transversal en prenant comme fil directeur la manière dont est appréhendée la dimension spatiale des conduites des jeunes. Par «dimension spatiale », on peut entendre l'articulation entre le social et le local, c'est-à-dire les modalités d'inscription sociale des jeunes dans les espaces urbains (marquage/neutralisation des territoires, enclavement/désenclavement, mobilité/sédentarité) et la diversité de leurs situations selon les configurations locales. Prenant acte des acquis de la sociologie de la jeunesse (diversité interne de la catégorie, report des seuils d'entrée dans la vie adulte, mutations intervenues dans le rôle de l'école, du travail social et dans le rapport à l'emploi), on fait l'hypothèse forte que ces travaux attestent des formes différenciées de l'inscription sociale des jeunes dans les espaces urbains.

La méthode proposée vient répondre aux difficultés posées à la fois par un découpage par thèmes à partir d'un ou de plusieurs critères selon leurs occurrences, et par une division du champ intellectuel des sociologies en fonction de courants dominants. Dans un cas, la difficulté réside dans l'isolement de thèmes, ainsi que dans l'analyse de leurs relations; dans l'autre, elle tient à la tendance à la dissémination des référents théoriques ou, à l'inverse, à la faible implication théorique de certains travaux. On propose en conséquence d'adopter une approche résolument diachronique consistant à retenir les travaux sociologiques les plus significatifs en étant particulièrement attentif aux effets de déplacement et à la récurrence des 


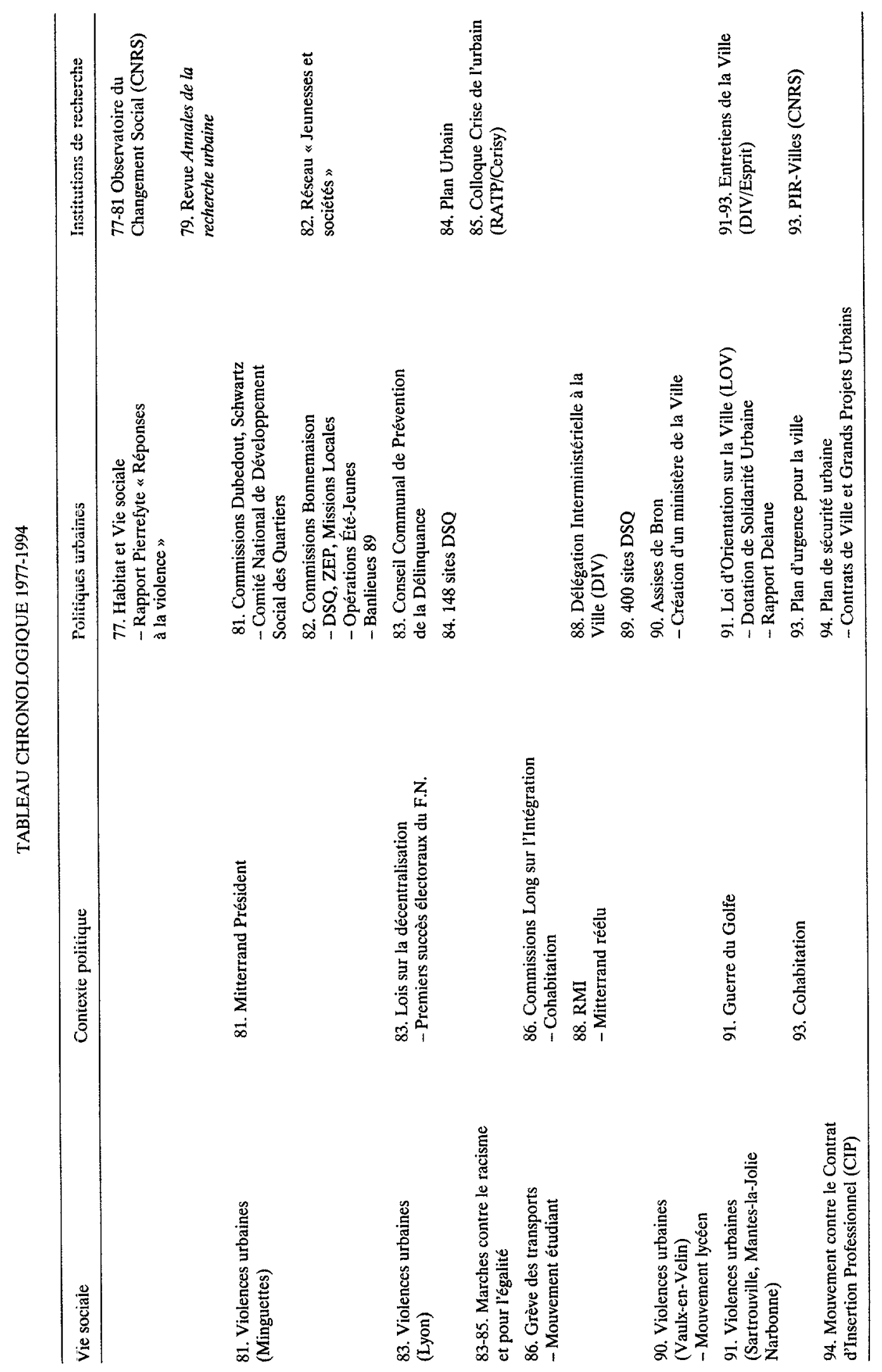


problématiques, aux continuités et discontinuités. Dans cette mise en perspective, on distinguera deux phases. Il a semblé opportun d'insérer un tableau multi-chronologique (voir tableau ci-joint) sélectionnant, de façon certes arbitraire, un certain nombre d'événements faisant sens par rapport à l'objet étudié, qui, regroupés en quatre rubriques, permettent dans une certaine mesure de justifier cette périodisation.

\section{BALISAGE DU CHAMP}

Il existe dans les pays anglo-saxons une abondante littérature consacrée aux phénomènes de délinquance et/ou de déviance juvéniles. Les formes de l'occupation de la rue par des bandes et leur fonction de socialisation y sont plus anciennes et mieux connues qu'en France, où domine la dissémination des réflexions et remarques. Outre les différences de contexte qui prévalent sur le plan de la structure sociale urbaine, sans doute pourrait-on expliquer ce phénomène comme un effet de la prévalence de la sociologie empirique aux États-Unis, et dans une moindre mesure en Angleterre, contrairement à d'autres pays comme l'Allemagne ou la France, où prédomine une approche critique. Il est d'ailleurs remarquable que ce thème ait été plutôt traité dans ce dernier cas par des travailleurs sociaux - ayant, pour l'occasion, étudié la sociologie ou s'y étant reconvertis - intervenant et mobilisant leurs compétences comme «professionnels de la rue ». Toujours est-il que c'est au tournant des années quatre-vingt qu’une série de déplacements conduisent à des travaux qui deviendront des « classiques» sur les jeunes des banlieues.

\section{I.1 GANGS, SOUS-CULTURES ET VIE URBAINE}

Aux États-Unis, le rapport «jeunes »/ «ville » s'inscrit dans le cadre de l'écologie urbaine. L'étude célèbre de F. M. Thrasher (1927) sur la formation des gangs et leur dimension territoriale montrait l'articulation entre les phénomènes de délinquance et les caractéristiques de la zone de transition selon le modèle de Burgess, le rôle de la composition ethnique des quartiers appelant selon leur homogénéité des réactions de solidarité et de défense, mais aussi le facteur constitué par les pratiques informelles des groupes d'enfants rendant compte du gang comme groupe conflictuel. Une caractéristique essentielle de cette formation était le mouvement, l'errance, la quête de nouvelles expériences. Ainsi, la variation de la fonction de conflit conduisait l'auteur à la construction d'une typologie des gangs intégrant leurs transformations. Analysant leurs conditions d'apparition et leur capacité de résistance aux forces de désorganisation, Thrasher illustrait l'idée selon laquelle le gang est une forme d'adaptation et d'organisation plutôt que de désorganisation dans un environnement donné. Il préfigurait ainsi les travaux de C. R. Shaw et M. D. Mac Kay (1940), et surtout de W. F. Whyte (1943) dans Street Comer Society sur la formation de micro-sociétés et les comportements de bande.

Dans les années 1950, l'hypothèse écologique est remise en cause par certaines théories de la déviance et la criminologie. Les critiques sont d'abord d'ordre méthodologique. S'appuyant sur des analyses statistiques, elles soulignent le manque d'explications autour de la répartition des phénomènes de délinquance ; l'absence de modèle de croissance urbaine et le problème d'une généralisation à partir de cas isolés; les difficultés à établir des corrélations entre zones de transition et comportement de bande ; le flou demeurant quant aux effets précis de diverses variables. Elles incitent ensuite à une analyse plus large des phénomènes de délinquance en minimisant les différences entre groupes de classe inférieure et de classe moyenne pour prendre comme objet l'adolescence dans une perspective anthropologique (Bloch, Niederhoffer, 1963), ou à l'inverse, en insistant sur les modes de socialisation différenciée selon les classes en termes d'anomie et de frustration au sens mertonien des termes (Cloward, Ohlin, 1960). Malgré ses déplacements, qui tendent à effacer la dimension territoriale des conduites déviantes, le gang n'en demeure pas moins un analyseur privilégié qui fait l'objet de dizaines d'ouvrages savants, parmi lesquels on peut retenir celui de M. Sanchez-Jankowski (1991) ayant pour originalité de porter sur une population de gangs ${ }^{4}$.

4. L. J.-D. Wacquant (1994) souligne que « le gang se situe aujourd'hui au point de convergence de plusieurs courants de recherche et de mouvements dits d'opinion. De sorte qu'il s'impose comme un sujet incontournable pour qui ambitionne d'éclairer les transformations contemporaines de la société et de la culture urbaines américaines ». 
En Grande-Bretagne, c'est dans les années soixante-dix que se développe une abondante littérature dont la singularité est d'être axée sur une sociologie des sous-cultures juvéniles. Dans un texte de 1972 considéré comme fondateur, P. Cohen (Butterworth, Weir, éds, 1980) explique que ce type de formation sociale s'est développé comme une réponse aux contradictions entre culture parentale, situation des jeunes sur le marché de l'emploi et rapports à la consommation de masse. Ainsi les groupes de teddy boys, mods ou skinheads constituent des sous-cultures qui s'inscrivent dans un contexte de déstructuration de la classe ouvrière et de marginalisation des nouvelles générations. À travers les modes de consommation (vêtements, musiques, alcool, drogues), l'identification à des territoires (le quartier, la rue, le stade de football) sont en jeu les valeurs traditionnelles d'une communauté ouvrière qui a cessé d'exister, ou la mise en scène d'une rupture avec cette condition (Hoggart, 1970). Dans ce cas, les significations spécifiques investies dans des pratiques ostentatoires sont définies comme " modes de stylisation et de symbolisation de l'existence » (Brake, 1980).

Parmi les critiques suscitées par cette approche, deux points peuvent être retenus : la tendance à majorer les capacités de résistance à la domination de ces sous-cultures juvéniles en passant outre leur caractère réactionnaire ; l'oubli des jeunes « respectables » qui, sans être des déqualifiés et des chômeurs, n'en commettent pas moins des actes délictueux (Mignon, 1991). C'est par la suite autour des phénomènes sociaux liés au football que de nouvelles approches se développent, prenant en compte les effets de structure et les diversités locales afin de rendre intelligibles les formes socialement différenciées de violence selon les opportunités du marché du travail, les « processus de décivilisation » (Mignon, 1991).

Par comparaison, les recherches menées en France sur le rapport « jeunes »/« ville » sont peu développées dans les années soixante et soixante-dix. Les travaux cités sont critiqués - quand ils ne sont pas passés sous silence ou méconnus. Si la découverte des bandes date de cette période, ce thème est très marqué par une « psychiatrie de l'adolescence ${ }^{5}$ ». Il donne lieu à quelques études ethnologiques dont la plus célèbre est celle, d'inspiration structurale, de J. Monod (1968). Pourtant, c'est lors de cette période de contestation étudiante et de crise économique qu'émergent les approches psychosociologiques et sociologiques de la jeunesse au travers des objets de recherche les plus divers et une grande dispersion thématique, comme le constate J. Jenny (Lagrée, Lew Fai, éds 1982). Mais dans un contexte intellectuel marqué par l'hégémonie du paradigme structuralo-marxiste, le thème du travail, le soupçon d'empirisme, l'essentiel des débats est ailleurs. Il est symbolisé par la querelle entre E. Morin et J.-C. Chamboredon qu'opposent deux conceptions de la jeunesse : comme classe d'âge et comme produit social de classe (Dubet, 1987 ; Galland, 1991). En rupture avec les illusions de mai 68, l'analyse du discours d'une population de 18-24 ans de milieux divers au début des années soixante-dix revèle une constante : « celle d'une recherche des niches, d'un repliement sur des bases privées, d'un enracinement dans des lieux privilégiés, abrités, isolés de la vie publique " (Duvignaud, p. 235, 1975 ). Mais il s'agit essentiellement de lieux symboliques (la moto, les bals, les amis, la musique...). Vers la fin de la décennie, un déplacement des interrogations s'amorce avec la publication de deux ouvrages « pionniers » : l'un porte sur les formes sociales de la marginalité (Mauger, Fossé-Poliak, 1977) ; l'autre sur le thème Les jeunes de la nue (Mury, de Gauléjac, 1977) qui traite du de la ville parmi d'autres sujets (les parents, le travail, l'argent, etc.).

Du côté des recherches urbaines, on sait que cette période est marquée par l'« âge d'or du marxisme urbain » (Amiot, 1986). C'est autour de la catégorie d'« équipements » qu'une part importante des travaux sur les jeunes se focalise pour rendre compte du fonctionnement et du rồle des appareils idéologiques de contrôle. Il s'agit de procéder à une approche critique de la conception selon laquelle l'absence de lieux spécifiques d'inscription tend à définir

5. Les deux préfaces de la traduction française de l'ouvrage de H. Bloch et A. Niederhoffer (1963) écrites par les docteurs S. Lebovici et P. Pichot en sont un indicateur, même si l'on pourrait interpréter cette double présentation comme un compromis entre deux conceptions de l'adolescence, l'une (déjà) psychosociale, l'autre (encore) médicale.

6. Voir aussi le premier numéro de la revue Autrement, «Jeunesses en rupture : dupes ou prophètes », 1/75 et A. Cauquelin (1977). 
socialement les jeunes comme marginaux, conception qui s'est concrétisée dans les années soixante sous la forme de lieux « adaptés » (centre social, maisons de jeunes, centres d'animation culturelle). Mais cette spatialisation du traitement social (Augustin, 1991) est confrontée à de nombreux effets pervers (désertion des structures existantes, dégradation, détournement d'usage). L'analyse des pratiques différenciées des groupes de jeunes, définies à travers la quête d'un lieu propre plutôt qu'assigné, en tant que rapports d'utilisation d'espaces fonctionnant comme spécification des positions sociales globales, invite à examiner le rôle d'une "ségrégation institutionnelle des jeunes » (Ferrand, 1974). Si par ségrégation on entend un « processus de production des pratiques spatiales », il s'agit alors moins de considérer des « groupes » que de comprendre les conditions sociales de regroupement et d'accaparement d'un espace défini comme « imminemment symbolique » (Ferrand, 1976).

Sur cette période, le constat établi dans un ouvrage de synthèse est tout à significatif (Lagrée et Lew Fai, éds, 1982). Il apparaît que cet objet constitue à peine un « chapitre du développement de la sociologie urbaine ». C'est tout d'abord la dissémination des remarques qui prévaut « lorsque l'illustration prend appui sur le problème des jeunes ou encore lorsque le problème de la cité, du quartier et de la ville soulève des questions que les jeunes posent aux responsables de la vie sociale » (ibid, p. 152). Ensuite, l'étude des communautés de vie et des groupes sociaux tend à l'emporter sur les rapports à l'espace. La vague d'urbanisation nouvelle conduit à des analyses du fonctionnement des quartiers, des logiques qui s'y inscrivent et des effets qu'ils produisent en termes de " transplantation résidentielle ». Les travaux de $\mathrm{H}$. Coing (1966), ou le fameux article de J.-C. Chamboredon et M. Lemaire (1970) permettent, certes, de rendre compte de la polarisation qui s'exerce sur les jeunes dans un contexte d'anomie et de changement social. Mais ils ne nous aident pas à comprendre leur place dans la ville. Or, remarque J.-C. Lagrée, on peut tenir pour acquis que la dimension spatiale joue comme facteur de l'identité et comme vecteur de différenciation sociale. Si l'espace n'est pas le lieu premier, prenant sens par la pratique, «l'investir en le codifiant, c'est se trouver positionné par lui, dans le système social de la ville $»$.

Contemporain des incidents survenues aux Minguettes qui inaugurent les « années-banlieues » (Jazouli, 1992), un tel constat est symptomatique du décalage qui existe entre certaines réalités sociales et les préoccupations des chercheurs ${ }^{7}$. La sociologie urbaine en particulier traverse une phase transitoire qui s'explique par divers phénomènes : baisse des crédits ministériels de recherche urbaine entre 1975 et 1984, intégration des « hors statuts » au CNRS, remise en cause des savoirs critiques, dispersion des référents (Lassave, 1994). Pourtant un vaste chantier est lancé entre 1976 et 1981, il s'agit de l'Observatoire du Changement Social, qui a pour enjeu de multiplier les études localisées et de prendre en compte les divers aspects des modes de vie (sociabilité, identité locale, pratiques associatives et culturelles), sans que la question des jeunes apparaisse de façon spécifique dans les seize volumes. On assiste à une relecture des textes des " pères fondateurs " (Halbwachs, Weber, Simmel), et surtout, à la traduction de textes de l'École de Chicago (Grafmeyer, Joseph, 1979). À partir de cette phase transitoire, l'essor de l'anthropologie urbaine est particulièrement significatif.

Si tournant des années 80 il y a, c'est dans la remise en cause des cadres de pensée aussi bien politiques et syndicaux que théoriques. Les effets de déplacement qui voient la ville se susbtituer à l'entreprise comme théâtre des conflits sociaux, les émeutes silencieuses (Vulbeau, 1992) aux mouvements sociaux, l'action publique à l'action politique «traditionnelle », d'une part, le discrédit des approches globales et des explications systématiques (Bachmann, 1993) et la sensibilité nouvelle manifestée à l'égard de l'objet local (Léonetti-Taboada, 1989), d'autre

7. On pourrait, à titre d'indice, comparer les actes d'un colloque tenu en 1985 - Année internationale de la jeunesse - jouissant de toutes les garanties tant institutionnelles que scientifiques (Les jeunes et les autres, CRIV, TI et II, 1986) avec le numéro d'une revue spécialisée (Les Annales de la recherche urbaine, "Jeunes urbains ès qualités", 1985). Si l'on prend comme critère les titres des communications, aucune ne fait référence à l' »urbain » ou à la « ville ». Pour ce qui est des auteurs, un seul apparait dans les deux cas - par la suite reconnu comme «le » spécialiste de sociologie de la jeunesse en France. 
part, conduisent à la constitution de problématiques nouvelles ${ }^{8}$. Celles-ci s'articulent principalement autour de trois pôles : les interférences entre les caractéristiques des quartiers dits « à risques » et les conduites d'auto-enfermement des jeunes confrontés à une période de latence ; la mise en place de « nouvelles politiques sociales territorialisées et globales » au sein desquelles ceux-ci occupent une place transversale (Dubet, Jazouli, Lapeyronnie, 1985 ; Duprez, 1989); les formes d'action et de mobilisation collective des "secondes générations " (Kepel, 1987 ;Lapeyronnie, 1987) qui s'opèrent parallèlement aux mutations du militantisme.

\subsection{LES JEUNES ET LA GALÈRE}

Deux ouvrages sont particulièrement significatifs du champ constitué et des cadres explicatifs proposés, d'autant plus qu'ils comportent un titre similaire : La galère (Lagrée et Lew Fai, 1985 ; Dubet, 1987). Traitant des conduites marginales et/ou de la marginalisation des jeunes des banlieues populaires, ils partagent le dessein d'en réactualiser la sociologie. Le terme même de «galère » est significatif d'une volonté ailleurs à l'œuvre de retrouver le point de vue de l'acteur et de valoriser le travail de «terrain » à partir de méthodologies originales (intervention sociologique pour l'un, analyse socio-biographique et indicateurs de groupes localisés pour l'autre).

L'ouvrage de F. Dubet propose une interprétation globale de la situation sociale des jeunes des banlieues populaires en termes de «crise du système d'action des sociétés industrielles ». L'auteur souligne d'abord les « logiques flottantes » de la galère, caractérisée comme une expérience où domine l'indétermination, la fragilité des réseaux, l'instabilité des situations. Irréductible à une logique unique (sous-culture de la délinquance, violence de classe, crise économique), elle s'organise autour de trois pôles (la désorganisation, l'exclusion, la rage), dont l'association conduit à la formation de logiques d'action (la protection, les combines, la violence sans objet). C'est la coprésence de ces logiques qui est originale, les oscillations collectives qu'elles permettent de situer, les ambiguités sur le plan des rapports établis avec les différentes catégories d'acteurs intervenant dans les groupes constitués. La galère ne se résume ni à un défaut d'intégration ni à un excès de frustration : elle ne se réduit pas à un mode de vie marginal ou à une sous-culture : elle est, par analogie plus qu'au sens propre, une « action de classe dangereuse ». Génératrice d'un climat latent de peur, sa singularité est d'être enragée.

L'analyse est ensuite développée à partir de deux axes. Le premier consiste à montrer que cette «expérience » de la galère est constitutive de la décomposition du monde ouvrier, en s'appuyant sur l'observation d'un groupe de jeunes dans une ville ouvrière traditionnelle de la banlieue de Liège. L'auteur souligne les différentes dimensions du « déclin des banlieues rouges" " à partir des récits et réactions de groupes d'adultes. Là où s'organisait un espace de vie autour de l'habitat, contre, tout contre l'usine, les quartiers dégradés sont beaucoup plus hétérogènes et leur population plus nombreuse et diversifiée. Quant au second axe, il conduit à mettre en relief le renversement qui se nourrit de la galère, définie comme « classe dangereuse », et comprenant les éléments d'une action de "classe laborieuse ». À partir de la situation des «jeunes immigrés » et de la lutte des Minguettes, il s'agit d'analyser les conditions d'émergence d'un nouvel acteur, mais aussi leur fragilité.

8. Voir par exemple F. Dubet et alii : Les opérations «été jeunes - Analyse longitudinale d'une politique publique, PUF-IDEF, 1986.

9. "La banlieue rouge, est, sur le plan local, la formation sociale qui résulte de l'interaction, autour d'un système politique municipal, d'une conscience de classe ouvrière et d'une communauté populaire fortement intégrée. " (Dubet, 1987, p. 219). En parallèle, S. Lazarus souligne aussi ce fait majeur : «En France, la catégorie de banlieue n'est réductible ni à un territoire (périphérie des grandes villes) ni à un peuplement (fortes proportions de chômeurs, de jeunes, présence et ascendance étrangère marquées...). Dans son usage actuel, c'est une catégorie récente, apparue il y a une dizaine d'années. Elle illustre une nouvelle conjecture de l'État consensuel et la fín des formes de conscience et de représentation en termes de luttes de classes. » $C f$. "Crise des banlieues, crise de l'État », colloque Ville, banlieue, lien social, Paris VIII, 12 et 13 janvier 1994. 
Outre le schéma analytique qu'il propose, cet ouvrage compte par la richesse des matériaux qu'il apporte sur l'univers social des ZUP, les modes d'existence des jeunes des classes populaires et leur perception par les groupes d'adultes et d'acteurs. Ainsi, certains des traits structurants de la vie des cités sont abondamment décrits : l'ambivalence du rapport à l'environnement, entre sentiment de pourriture et logique de la solidarité, l'attachement au quartier opposé au racisme du dehors, la chaleur des relations opposée à l'indifférence de l'environnement, la revendication d'un local ou la capacité des sujets à devenir acteurs locaux, la sociabilité en réseau et l'occupation des espaces communs. En cela, il n'est pas étranger à une sociologie de la ville. Cependant, on peut regretter que l'espace urbain se réduise à un « décor», évoqué comme «paysage » dont le changement a valeur d'indicateur de transformations plus profondes. Les enjeux spécifiques du territoire des cités, les ressources qu'il offre sur un plan social, économique et symbolique sont peu abordés (Dubet et Lapeyronnie, 1992). Parmi les critiques adressées à cet ouvrage, certaines ont souligné la forte localisation de l'enquête à la région parisienne, à l'exception des Minguettes et de Seraing qui sont des sites tout à fait spécifiques, ce qui laisse entière la question des variations des logiques repérées selon les situations locales (caractéristiques des quartiers, histoire locale ou régionale).

L'autre ouvrage sur la galère part de la notion de marginalité : si elle fait sens, sans se réduire au fonctionnement du système social, encore faut-il se donner les moyens d'en rendre compte à partir d'une démarche « compréhensive », avancent J.-C. Lagrée et P. Lew Fai. Sinon se dérobe le processus de «micro-différenciation symbolique » sous-tendu par les rapports sociaux. Dans cette perspective, la capacité des acteurs à moduler, réinterpréter, transformer les normes sociales, les enjeux des logiques d'action et de réaction des bandes, les effets qu'elles produisent et les arrangements qu'elles rendent nécessaires au sein d'une collectivité sociale, sont au centre de l'analyse. Tout l'ouvrage souligne cette dialectique des rapports entre effets de structure et actions sur la structure, assignation d'une position sociale et promotion d'une identité propre, appartenance de classe et modèles culturels. Cette démarche est rendue opératoire par l'étude des procès d'intégration et de marginalisation à partir de la « collectivité résidentielle ", considérée comme le dernier lieu où les jeunes cherchent à se valoriser et s'exposent à la relégation. Elle se déploie principalement de la façon suivante : après avoir analysé les multiples facettes et les déterminations structurelles de l'implication des jeunes dans leur univers résidentiel en les rapportant à leurs trajectoires socio-biographiques, puis examiné les réaménagements commandés par la quête et l'obtention de la reconnaissance sociale dans le cadre des rapports intra et inter générationnels, les auteurs abordent les « effets de la dynamique produits par ces différentes interactions sur la manière dont les jeunes perçoivent et recomposent leur positionnement local » (Lagrée et LewFai, p. 154).

Les mécanismes qui participent à la constitution d'une collectivité sont particulièrement soulignées - quoique l'on puisse regretter que les trois «terrains » étudiés, dont une ville nouvelle, soient en région parisienne. Ainsi en va-t-il des modes de regroupements qui varient en fonction de la durée d'implantation dans le quartier, de l'âge du changement de résidence, de la composition sociale des espaces de vie. Le positionnement des jeunes dans le jeu social local prendra telle ou telle forme selon la spécificité de l'environnement, sa structuration interne et l'importance des rapports de classe. Ce qui amène à distinguer la manière dont l'identité sociale se constitue en milieu homogène ou hétérogène, l'« effet du local " sur les trajectoires. Les auteurs montrent aussi que le rapport des membres des groupes et bandes à leur environnement ne passe pas seulement par des composantes matérielles mais par les références symboliques qui contribuent à un marquage social de l'espace.

Ces deux ouvrages établissent donc que les jeunes de la « galère » appartiennent à la fraction précaire de la classe ouvrière et qu'ils sont eux-mêmes plus exposés aux risques d'exclusion sociale - phénomène interprété comme plus ou moins accentué chez les jeunes d'origine étrangère -, mais soulignent de façon complémentaire le rôle de l'espace urbain dans la construction des identités. 


\section{I.3 LES QUARTIERS, ESPACE ANOMIQUE OU TERRITOIRE CONSTITUÉ ?}

La lecture misérabiliste qui en a été parfois faite pose un problème général : peut-on penser les grands ensembles et les cités des banlieues populaires comme un espace social de type anomique, au regard de la transformation de sa stratification et de la masse d'exclus qui s'y concentre, ou bien peuvent-ils être aussi analysés dans leur diversité comme un espace constitué avec ses territoires, ses normes de référence, ses mécanismes de régulation propres? Dans la mouvance des investigations ethnologiques de C. Pétonnet (1979 et 1982) ou G. Althabe (1984), la multiplication des monographies dans le champ urbain sur les milieux défavorisés vient donner consistance à cette alternative ${ }^{10}$. Ainsi ces travaux participent-ils à la redéfinition du rapport des groupes sociaux aux espaces urbains à partir d'un second pôle de recherche.

L'enquête de terrain réalisée par C. Calogirou (1989) au sein d'une ZAC de la région parisienne est tout à fait exemplaire à cet égard. Elle a pour objet d' « appréhender la logique qui articule les rapports sociaux internes au champ résidentiel $»$. Elle montre que c'est autour du principe de ragot que réside cette articulation. Dans un univers d'homogénéité sociale, le ragot est compris comme «processus de déclassement des autres/surclassement de soi ». Il s'inscrit dans une logique typiquement méditerranéenne de l'honneur, qui prévaut dans des sociétés masculines. C'est bien ce qui distingue le ragot de la rumeur et du commérage : portant atteinte à l'honneur de la famille, il participe à la structure des rapports sociaux. Au sein de ce processus, les jeunes (en particulier maghrébins) occupent un rôle de médiateur de par leur caractère visible/invisible dans l'espace résidentiel. Leur occupation des parties communes ou de la rue, support de socialisation, apparait comme l'expression à la fois de certains problèmes sociaux (drogues, délinquance, alcool) et des capacités éducatives des familles. En fonction de ce principe et des places qu'il implique, C. Calogirou rend compte de plusieurs catégories de pratiques des adolescents selon qu'ils se situent dans le ragot (stationnement), le fuient (mouvement), ou sont dans un lieu neutre, selon qu'ils s'y assimilent ou s'en distancient. Elle analyse aussi le rôle - variable - des filles, investies de l'honneur de la famille et des pratiques de compromis entre le réseau familial et celui des copains.

Ainsi les stéréotypes sur l'anonymat des grands ensembles sont mis à l'épreuve par l'analyse du ragot, considéré comme mécanisme de régulation interne qu'actualise le jeu constant des embrouilles, des procès d'intention et du rappel à l'ordre au nom du respect. Quant à l'espace, il apparaît comme un enjeu symbolique fort, de par le rôle de la sphère publique comme support essentiel de la vie sociale. Du coup la question se déplace, elle devient celle des possibilités de généralisation d'une approche localisée sur un quartier. Les mécanismes décrits dépendent-ils de conditions socio-morphologiques qui en attestent les limites ? Pourtant, on pourrait multiplier les références d'enquêtes révélant le fonctionnement « communautaire » des cités, les interactions entre sociabilité de quartier et socialisation familiale dans l'exacerbation du sentiment des jeunes maghrébins d'appartenir à une «communauté de destin résidentiel » (Blöss, 1989), le rôle des associations et des médiateurs/formateurs dans l'élaboration d'un espace public local (Joseph et alii; Anselme, Péraldi, in Les Annales de la recherche urbaine, 1985).

\section{VERS UN RENOUVELLEMENT DES APPROCHES}

Comment situer les travaux du début des années quatre-vingt-dix ? Sans majorer les effets de rupture que l'on pourrait attribuer à un manque de recul, ni les continuités qui pourraient être mises sur le compte d'effets de méconnaissance, on peut poser que trois caractéristiques sont à l'œuvre, qui soulignent les interférences entre le contexte, les centres d'intérêts et les approches. On assiste à une aggravation de la situation dans les quartiers dits « sensibles » qui cristallisent les processus de transformations sociales ailleurs à l'œuvre. Cette situation,

10. Il y aurait beaucoup à dire sur les différences entre ethnologie dans et de la ville, les apports et limites de l'ethnologie du « proche " à la compréhension des sociétés urbaines et au renouvellement des approches qualitatives, le rapport au « terrain». Voir notamment M. Clavel, 1992. 
qui s'accompagne d'une intense mobilisation des pouvoirs publics, incite à frayer de nouvelles pistes de recherche. Ainsi une diversification des centres d'intérêts voit le jour, doublée d'une globalisation du discours sur les banlieues. Pourtant, le constat des limites d'une approche localisée tant sur le plan théorique que pratique conduit à un déplacement des lieux et des situations observés. C'est en cela que résiderait un renouvellement de l'approche socio-spatiale des jeunes marquant un refus de la sociologie de la déviance.

\section{II.1 LA CONSTRUCTION SOCIALE D'UNE IMAGERIE DES BANLIEUES}

Dix ans après les « rodéos des Minguettes ", la mort d'un motard par suite d'une poursuite avec la police déclenche des incidents trois jours durant. Ce que l'on appelle les « émeutes de Vaulx-en-Velin" marquent le réveil des banlieues. Entre ces deux repères devenus mythiques se déploie l'histoire immédiate et discontinue de la " France des banlieues" ». Cette histoire se déploie d'après des temporalités variables selon les acteurs et les institutions. D'un côté, le temps est à la gestion de l'urgence : bilans, groupes de pilotage, rapports « officiels ${ }^{12}$ " se multiplient, alimentent la presse et ses chroniques alarmistes sur les menaces d'explosions sociales, la violence, la drogue. On retrouve à l'œeuvre le cycle bien connu effets d'annonce/ promesses/subventions/frustration. Il est d'un autre côté à la compréhension dans la durée et à la comparaison dans l'espace ${ }^{13}$. En schématisant, trois lignes de force organisent la production sociologique : l'analyse des processus socio-politiques et médiatiques, y compris à travers la comparaison d'expériences nationales sur le plan des politiques publiques et des logiques d'action; l'analyse des propriétés structurales dont les logiques macrosociologiques produisent des effets qui ne cessent de défaire ce que les acteurs locaux tentent de faire ; l'analyse de la multiplicité des configurations locales en fonction du traitement institutionnel, des stratégies municipales et de leur emprise sur la vie sociale des cités.

Pourquoi tant d'émoi ? Ces incidents symbolisent tout d'abord les limites d'une gestion politique des banlieues (Body-Gendrot, 1993). Sont mises en cause les formes d'intervention publiques mises en place dans les années 80 , la « politique de la ville » sur lesquelles elles ont depuis peu débouché (Donzelot, 1991 et 1994), mais aussi les contraintes rencontrées dans la gestion locale de la «crise sociale » par les divers acteurs sociaux et institutionnels (Bailleau, Garioud, 1990 ; Duprez, Heddli, 1992). Si Vaulx-en-Velin a cristallisé l'attention publique pour, de petit bourg qu'il était devenir symbole de la « crise des banlieues », c'est qu'il constituait une vitrine des opérations de réhabilitation et de développement social (Linhart, 1992). Mais l'explosion de 1991 traduit aussi l'accumulation des malendendus, et en particulier le peu de place accordé aux questions d'ordre public au profit des thèmes du chômage et de l'insertion là où les tensions avec la police sont pourtant au cœur de l'expérience des jeunes des cités (Battegay, Boubeker, 1991 ; Lapeyronnie, 1993). À cette dimension politique et institutionnelle s'associent ensuite de nouvelles logiques sociales. Logiques d'action des jeunes émeutiers dont la « rage » est de plus en plus alimentée par un sentiment de mépris et d'injustice. Logiques identitaires, voire territoriales de groupes qui affirment de plus en plus leur appartenance à une communauté maghrébine et musulmane. Enfin, il y a le travail d'amplification et de mise en scène de la presse et de la télévision : lorsque la logique médiatique s'emballe, les

11. Voir les numéros spéciaux de revues, « La France des banlieues », Esprit, février 1991 ; « Banlieues, relégation ou citoyenneté », Les temps modernes, $\mathrm{n}^{\circ}$ 545-546, déc. 1991-janv. 1992, "Jeunesses et sécurité », Les Cahiers de la sécurité intérieure, $\mathrm{n}^{\circ} 5$, mai-juillet 1991 ; voir aussi Ethnologie française, "Violence, brutalité et barbarie ", 1991, 3.

12. Voir, J. M. Delarue, Banlieues en difficulté : la relégation, Paris, Éd. Syros, 1992; A. Jazouli, Jeunes des banlieues, violence et intégration : le dilemme français, FAS, 1991 ; J. Dray, Sur la violence des jeunes dans les banlieues, Commission des Affaires culturelles, familiales et sociales, Assemblée nationale, 1992.

13. En même temps, ce clivage est moins net qu'on veut bien le dire. Si la division du travail social impose une partition bien établie des registres d'intervention, quant aux effets (domination, stigmatisation) et contre-effets (dédramatisation, relativisation) qu'ils engendrent, la « demande sociale » incite à la confrontation des points de vue entre les différents acteurs (politiques, gestionnaires, professionnels, chercheurs). Fait capital, il se produit une certaine confusion des genres de discours, manifeste à travers la superposition des catégories politico-administratives, journalistiques, scientifiques qui mériterait d'être analysée de façon systématique. 
incidents deviennent « émeutes », les violences des « explosions sociales », les groupes de jeunes «blacks » ou « beurs » des « bandes ethniques », les faits divers des « phénomènes de société ", etc. C'est sans compter les effets ambivalents de stigmatisation et de reconnaissance que produisent les médias, leur détournement pour les « casseurs » afin d'accéder à une visibilité minimale, pour se faire entendre.

La compréhension des processus en jeu passe par un travail critique vis-à-vis des stéréotypes constitués (banlieue, ghetto, bandes, ethnies...). Ainsi des travaux d'inspiration diverse ont pour trait commun l'analyse des mécanismes de construction sociale d'une imagerie des banlieues et des bandes de jeunes. Si par « imagerie " on peut entendre la sélection de situations emblématiques incarnant des mythes en fonction d'un marquage territorial (Bachmann, Basier, 1989), l'Amérique en constitue un réservoir inépuisable. Autour de la notion de "ghetto " s'est constituée toute une polémique. Les travaux comparatifs montrent que, quels que soient les indicateurs retenus (ségrégation, pauvreté, criminalité, etc.), les réalités sociales de part et d'autre de l'atlantique sont sans commune mesure (Body-Gendrot, 1993). Des facteurs de convergence apparaissent ici et là (dépopulation, échec scolaire et chômage accentué, etc.), mais on ne peut confondre logiques de ségrégation ethno-raciale et formes d'exclusion socioéconomiques qui différencient le ghetto noir américain des banlieues françaises. De même, le désengagement de l'État fédéral, qui rend compte du passage d'un ghetto communautaire à un « hyper-ghetto », tranche avec la construction politique de la ville (Wacquant, 1992 et 1993 ; Vieillard Baron 1994). On pourrait faire la même analyse à propos du « retour des bandes ». De nombreux articles ont souligné les amalgames entre gangs, bandes et sociabilité amicale. Confrontant effets de croyances journalistiques et policières d'une part, constats des acteurs de terrain d'autre part, A. Jazouli (1992) a mis en relief l'accentuation d'un processus de désagrégation du lien social se manifestant par la multiplication de quasi groupes au caractère informel et éphémère.

Si le rôle des médias occupe une place centrale dans la construction sociale de ces imageries, il a peu été abordé de façon systématique. Outre l'étude de F. Portet (Dubet et al, 1986) et celle de Bachmann et Basier sur La Courneuve, on mentionnera parmi les travaux les plus récents deux approches. Pour l'une, il s'agit de montrer en quoi la presse et surtout la télévision fabriquent une représentation de la réalité des faits, un effet de réel (Champagne, 1993). Cette construction renforce la prégnance des représentations dominantes et les stéréotypes sur les banlieues tristes et dangereuses. Loin de profiter aux plus démunis en capitaux économiques et culturels, ce travail conduit à redoubler leur domination dans la mesure où les habitants de ces quartiers stigmatisés intériorisent et tendent à « réciter » ce discours (Mauger, 1992). Une telle circularité témoignerait des profits médiatiques en jeu et du primat des luttes symboliques qui structurent le champ politico-journalistique ${ }^{14}$. Pour l'autre, il s'agit de mettre en relief le «travail de figuration " et les «modes de publicisation " de l'immigration dans l'espace public (Battegay, Boubeker, 1994). De la Marche des beurs à l'Affaire des foulards, des images publiques sont construites, corrigées, réglées sous l'action de multiples acteurs, jusqu'à constituer parfois un enjeu prioritaire de l'action, rethématisées en fonction du contexte. L'importance de la presse locale, le lien entre la « montée en affaires » et le calendrier politique, l'inscription de ces événements dans l'espace public, sont les principales caractéristiques de cette médiatisation. Mais ce qui est le plus remarquable, soulignent les auteurs, ce sont la "centralité épisodique du thème de l'immigration dans les débats publics et le positionnement périphérique du dossier de l'immigration dans l'action publique. »

\section{II.2 SOCIOLOGIE DES QUARTIERS ET EXCLUSION}

Si les interférences entre texte et contexte sont manifestes dans les thèmes abordés et leur traitement par les chercheurs, elles s'accompagnent d'un mouvement contradictoire. D'un

14. Il serait intéressant d'analyser l'usage des reportages sur les « banlieues », par exemple dans le sens d'un renversement du stigmate. Voir à ce sujet l'analyse des stratégies d'images et de rétention d'informations déployées par les membres des gangs américains vis-à-vis des journalistes et les bénéfices qu'ils en attendent (Sanchez-Jankowski, 1994). 
côté, il se produit en effet une diversification des centres d'intérêt et des thématiques. On songe en particulier aux travaux déjà rencontrés sur les territoires des bandes, les stratégies et trajectoires résidentielles, les politiques locales de la jeunesse, l'immigration, mais aussi sur les représentations et les usages de l'environnement, la production symbolique et les univers culturels, l'usage et le trafic de drogues illicites. D'un autre côté, on constate une globalisation du discours que symbolise la notion d'« exclusion ». La thématique de la relégation (spatiale, scolaire, professionnelle), puis celle de l'exclusion (sociale, culturelle, politique) désigne cet ensemble de phénomènes qui traduisent ou sont le symptôme de transformations sociales globales (Touraine, 1991). Elle suggère en quelque sorte une représentation qui fait défaut à une société post-industrielle confrontée à une crise des modes d'identification et d'appartenance collectives. Il en résulte que le rapport « jeunes »/ »ville » est à la fois tout à fait central de par l'accent mis sur la localisation des «problèmes sociaux », et périphérique de par son caractère éminemment symbolique qui fait référence à un ordre de considérations plus général.

L'analyse des mécanismes de régulation sociale dans les quartiers permet de concrétiser un tel schéma. Si les flambées de violence ont été souvent imputées à la dégradation du cadre bâti, ce lien n'a rien de «mécanique». On sait que ce n'est pas dans les quartiers les plus dégradés et marginalisés que les actes de révolte et d'autodestruction se produisent ${ }^{15}$. Entrent en jeu des phénomènes complexes d'identification et d'appartenance collectives qui conduisent à éviter l'isolement de certaines enclaves dans la ville. D'autres facteurs explicatifs interviennent aussi ; certains sont relatifs à la précarité relationnelle, à la vulnérabilité des modes de vie, à la crise de la mobilité sociale (Laé, 1991); d'autres ont trait à la substitution des services sociaux aux anciennes sociabilités ouvrières, des régulations de contrôle aux régulations autonomes (Dubet, Lapeyronnie, 1992). Dans les deux cas, il se produit une recomposition des identités sociales : faute d'identités de condition (ouvrière) ou d'identités de position (salarié, citoyen), certains des jeunes s'inventent des identités de lieu (Kokoreff, 1994). Toutefois, ce processus ne produit pas les mêmes effets partout ${ }^{16}$. De plus, il est nécessaire de distinguer entre identité de quartier et espace identitaire. Si les effets de la désagrégation sociale dévalorisent l'une, les modes d'appropriation des espaces publics et semi publics, la focalisation sur les emplois de quartier favorisent l'autre (Bouamana, 1993).

La transformation des modes de régulation des quartiers pose aussi la question de la cohabitation et de l'ethnicité urbaines. Selon les uns, alors qu'ils sont traversés par une dynamique conflictuelle entre insularisation et irrigation, on assiste à la mise en place d'un mode de « régulation sociale ethnique » (Begag, Delorme, 1994). C'est l'introduction du principe de discrimination positive dans l'embauche de chauffeurs de bus, de vigiles, d'animateurs, de surveillants, d'enseignants de type «maghrébin » ou « noir-africain ». C'est aussi le rôle de l'islam qui fonctionne comme agent de prévention interne face à l'échec des politiques locales. Mais cette «thèse » peut être nuancée. Des enquêtes quantitatives, comme celles réalisées sous la direction de M. Tribalat (1995), montrent bien les limites de la sociabilité communautaire à laquelle échapperaient la plupart des groupes. Une enquête qualitative menée à Marseille indique que les logiques d'acteurs à l'ouvre dans l'espace public local ont plus pour enjeu l'attachement au quartier, à une classe d'âge exclue ou la professionnalisation d'un milieu intermédiaire que des références d'ordre communautaire - ce qui n'empêche pas la

15. Les données du dernier recensement (1990) permettent de caractériser les « quartiers prioritaires » à partir de trois indicateurs : un taux de chômage $(19,7 \%)$ près de deux fois supérieur à la moyenne nationale $(10,8 \%)$, concernant $24 \%$ des hommes et $34 \%$ des femmes de $20-24$ ans ; une surpopulation juvénile $(32,9 \%$ des habitants avaient moins de 20 ans, contre $26,5 \%$ en moyenne) ; la concentration de familles étrangères (18,3\% contre $9 \%$ pour les agglomérations concernées). Ces données font aussi apparaître des situations contrastées sur le plan régional en ce qui concerne les formes urbaines (à la différence de la région parisienne, $58 \%$ des quartiers présentent, par exemple, un tissu urbain composé d'immeubles et de pavillons) et le taux de chômage (14,4\% en île-de-France, $16,7 \%$ en région Rhône-Alpes, 25,3\% dans le Nord-Pas-de-Calais). Cf. M. Castellan, M. Marpsat, M. F. Goldberger, « Les quartiers prioritaires de la politique de la ville ", INSEE Première, n" 234, décembre 1992.

16. Si, par exemple, dans le cas des quartiers d'habitat social en région parisienne, on constate une quasiabsence d'identification ouvrière, les situations par ailleurs observées dans les ZUP de l'agglomération lilloise condujsent à nuancer ce constat. 
mobilisation de ses ressources, des stratégies de « mise en scène ethnique » afin d'obtenir des subventions (Césari, 1994). Selon d'autres travaux, le fait nouveau est que les jeunes se définissent et sont définis comme les membres d'une «ethnie ». Mais l'ethnicité en jeu ne se confond ni avec la culture d'origine ni avec l'existence d'un espace communautaire : elle est une production symbolique bricolée à partir d'emprunts divers (Roy, 1993). Le problème est moins celui de l'immigration que celui de l'éclatement de la communauté d'origine maghrébine. Il est incarné par une minorité acculturée mais non intégrée, par la petite délinquance et les incivilités qu'elle implique, particulièrement dans les moyennes petites et moyennes villes de province confrontées à l'interpénétration des milieux sociaux et des micro-communautés dans les établissements scolaires, la rue, les boîtes de nuit.

L'accentuation des situations de précarité par rapport à l'emploi pose aussi la question du fonctionnement d'une économie informelle dans les quartiers (Bachmann et Coppel, 1991; $\mathrm{CNV}, 1994)$. On commence à mieux connaître, en France, les conditions sociales de diffusion des drogues illicites, et notamment les systèmes d'échange et d'entraide qui constituent des connexions entre solidarité de quartier et marché, la différenciation et la plasticité des types d'investissement et de contrôle des « réseaux de cités ", les contreparties à la dévalorisation trouvées du côté du partage des risques (Bouhnik, Joubert, 1992). Ces travaux et d'autres en cours indiquent bien les enjeux de la notion de territorialité : il s'agit saisir l'assise des usages et trafics, la superposition de différents registres du lien social (sociabilités familiales, amicales, de quartier, services informels ou activités économiques) ; mais aussi de repenser l'échelle d'intervention des phénomènes en raison des mobilités et des interdépendances qu'impliquent modes de consommation, de solvabilisation et d'approvisionnement.

Ces analyses posent de façon le plus souvent implicite la question des modes de perception et de représentation de l'espace urbain. Toute une tradition de recherches urbaines a développé le thème des "villages dans la ville " que monde des ZUP incarne à sa manière. Certains travaux ont été menés sur les variations des perceptions selon les populations (jeunes/adıltes, métropolitaines/non métropolitaines), entre l'image du ghetto et celle des HLM (Vieillard-Baron, 1990), d'autres encore sur la perception des contours des quartiers, en soulignant leur existence « toujours de l'autre côté, plus loin, dans le miroir de sa réputation » (Wittner, 1992). Dans une perspective plus systématique, G. Mauger (1994) propose de prendre pour objet les représentations et les usages de l'environnement de différentes catégories de jeunes issus de milieux populaires, de mettre en rapport leurs visions du monde social et leurs dispositions à l'égard du monde dans lequel ils vivent avec leurs différentes trajectoires (familiales, scolaires, résidentielles, professionnelles). Distinguant les différentes cartes de l'« espace social vécu » et de l'« espace social imaginé », il propose plusieurs hypothèses explicatives de leur morphologie, des critères de classements des agents et des lieux et des dispositions de différentes catégories de jeunes.

\section{II.3 AU-DELÀ DE LA SOCIOLOGIE DE LA DÉVIANCE}

Longtemps, le rapport entre ville et jeunesse a été informé à partir des représentations de la dangerosité sociale de la « rue ». Or cette situation s'avère anachronique au regard des mutations actuelles de la jeunesse et des modes de fréquentation des espaces publics urbains qu'elles impliquent : sociabilité tournée vers l'extérieur, crise des institutions de socialisation, devoir d'insertion, défaut ou inadéquation des équipements collectifs et institutionnels (Barreyre, Vulbeau, 1994). Mais, pour prendre toute sa pertinence, cette démarche implique une double rupture : avec le « localisme » qui confine dispositifs publics et pratiques urbaines à une échelle réduite, et avec la stigmatisation des pratiques de la ville et des jeunes présupposés déviants. Il est donc nécessaire de disposer d'outils conceptuels qui permettent de penser la dynamique des rapports entre espace et socialisation, urbanité et sociabilité, comportements culturels et territoires urbains. Ces outils, c'est principalement le renouveau de l'anthropologie urbaine qui va les fournir, à travers notamment la notion d' " espaces publics » et d'accessibilité » (Joseph, 1984, 1994), l'analyse des formes de mobilité proprement urbaine, (Grafmeyer, 1994 ; 'Tarrius, 1994). 
S'il existe d'innombrables monographies de quartiers, plus rares sont les travaux portant sur les pratiques de mobilité. Certains mettent en relief l'organisation sociale différenciée de ces pratiques ; la ritualisation des déplacements et ce qui s'y joue pour une population vouée à une quasi-absence de projets de vie ; l'importance d'« espaces publics stables » qui n'ont pas à être négociés quotidiennement et donnent le sentiment qu'il existe des endroits dans la ville où ils ne sont pas marginalisés - rappelant ainsi que l'espace confère sa matérialité au groupe (Vidal Naquet, 1985). D'autres soulignent davantage les tensions de l'immobilité et de la mobilité constitutives des modes de vie des familles immigrées, ainsi que la diversité des formes de « sortie " (Begag, 1991). Particulièrement intéressants sont les travaux menés à partir de la tension centre/périphérie, mobilité/insertion ; ils attestent du rôle des formes de l'espace urbain dans le processus d'intégration des jeunes; ils montrent les enjeux de l'accès à l'espace public et leurs différentes dimensions (mythique, initiatique, identitaire, transitoire). Ainsi la mobilité peut être un facteur de socialisation, là où l'insertion est d'ordinaire pensée sous forme de dispositifs territorialisés (Forêt, Payet, 1986).

L'émergence de diverses pratiques urbaines telles que les graffiti qui ont investi les espaces urbains des grandes villes (Kokoreff, 1991 ; Vulbeau, 1992), l'engouement pour la musique rap et le monde du « hip hop » ou des " zoulous » (Rousselot, Lapassade, 1991 ; Guidicelli, 1991), les sports de glisse (skate-board, roller-skate) et les consommations y étant associées (Fize, Touché, 1992), est significative de la spécificité juvénile de la sociabilité. Il s'agit d'être ensemble, d'investir des lieux pour être, de se donner à voir. Mais il s'agit aussi de défi, de mobilisation de compétences propres, de mise en relation. C'est en cela que ses pratiques peuvent être dites « urbaines » : inséparables d'une dimension spatiale (la rue, le métro, l'esplanade) qui leur confère une visibilité sociale, elles s'appuient sur des formes d'action collective qui font les jeunes des acteurs potentiels d'un espace public (Vulbeau, 1994). Si elles mettent en scène différentes catégories sociales de jeunes avant d'être attribuées en propre à telle ou telle, c'est qu'elles soulignent leur position originale en tant que génération dans l'espace social qu'une vision en termes d'anomie ou d'exclusion contribue à occulter (Battegay, 1985).

La démarche proposée par L. Roulleau-Berger est significative d'une tentative de penser et de concilier ces différents plans d'analyse (mobilité, cultures urbaines). Ainsi la notion d'espaces de transition culturels vise à comprendre les ajustements entre aspirations sociales et ressources offertes par les marchés du travail face au renforcement des situations d'instabilité professionnelle et de fragilité statutaire d'une partie croissante des jeunes (Roulleau-Berger, 1991). Si la ville est un « univers des possibles », elle favorise la constitution de projets, de sociabilités en réseaux, de modes de vie alternatifs, échappant à l'instrumentalisation et au contrôle, par stratégies et par ruses. Autrement dit, ces espaces ont ceci de spécifique qu'ils autorisent une intermédiarité statutaire autant que physique ; ils participent à la création de lieux de mise à distance de l'emploi et de proximité de groupes de jeunes socialement et culturellement hétérogènes, de production de cultures et d'évitement des politiques urbaines. Ces « espaces intermédiaires » sont aux frontières des espaces institués et des sources de régulation sociale plus ou moins autonomes. Par là, il s'agit d'échapper au « stéréotype stigmatisant de la marginalité » afin de prendre en compte les échanges entre les jeunesses, de rompre avec une sociologie dominée par les théories de l'étiquetage pour constituer une sociologie des intervalles (Joseph, 1984). Il ne s'agit pas de se cantonner à une approche localisée mais de poser la question d'une « recomposition d'un ordre spatial » dont l'éclatement des rapports centre/périphérie est le signe le plus manifeste. Dans un article récent, l'auteur prolonge cette perspective théorique entre ville et emploi en proposant un certain nombre d'outils conceptuels qui visent à éclairer de façon nouvelle les phénomènes de précarité et de chômage. Elle évoque la constitution de "cultures de l'aléatoire " dont les jeunes en situation précaire sont le symptôme ; souligne comment elles peuvent évoluer vers des " cultures de l'urgence » à travers la figure des sans-abri. En cela, il s'agit de substituer aux notions flottantes d'insertion ou d'exclusion les outils afin de penser une diversité de situations (Roulleau-Berger, 1994).

Dans une perspective comparable, on peut formuler l'hypothèse selon laquelle le rapport à l'espace des jeunes constituait une des dimensions de leurs différenciations sociales (Kokoreff, 
1993). Prendre en compte leurs expériences urbaines, c'est considérer des pratiques d'espace en tant qu'elles sont le produit d'une tension variable en intensité et dans leurs formes entre immobilisation forcée et désir de mobilité. En fonction de groupes donnés, on peut mettre en relief une configuration spécifique entre territorialité et mobilité. L'expérience urbaine des jeunes des cités oscille entre une accentuation de la sédentarité et l'investissement de microrepaires dans la ville. Plus leur marginalité est forte, plus cette tension devient socialement problématique pour les institutions et les acteurs en jeu (gardiens, policiers, agents de transports...). D'où le potentiel à la fois d'identité et de violence dont ces territoires sont porteurs, les dégradations dont le réseau de transport - comme espace transitoire - est l'objet. L'expérience urbaine des jeunes « alternatifs », d'origine sociale plus diversifiée et impliqués dans des associations, groupes de musiques ou autres, est centrée sur des « lieux de vie » (squats, locaux) et un réseau de villes et de relations qui définit une aire culturelle manifestant une meilleure maîtrise de leur environnement et de ses ressources. Celle, enfin, des jeunes issus de la petite-bourgeoisie et de la bourgeoisie locale, est plus axée sur les lieux privés - éventuellement squattés lors de «soirées »- et les équipements de consommation de masse (cinéma, fast-food).

\section{CONCLUSION}

On se demandait quelle était la pertinence de la dimension spatiale des conduites des jeunes. En cherchant à souligner la singularité du cas de la France, on a vu comment cette question s'est progressivement constituée, au tournant des années quatre-vingt, au carrefour de plusieurs approches. Deux pôles sont ressortis tout particulièrement pour s'inscrire : une sociologie de l'action cherchant à renouveler la sociologie des conduites marginales; une ethnographie des enclaves urbaines participant à la recomposition de la réflexion sur la ville. Puis, on a mis en relief la façon dont la spatialisation de la question sociale, à travers la figure des «banlieues ", a débouché sur un ensemble de travaux signalant le rôle central de la «population jeunes " au sein de ces processus socio-politiques, mais aussi la prégnance du lieu des conduites et représentations de certaines catégories de jeunes, les articulations complexes entre groupes de pairs, territorialité et mobilité. Par là ressortent les enjeux spécifiques d'une approche en termes d'inscription spatiale des phénomènes sociaux; et ce, sans en rester à l'analyse des territoires résidentiels et des logiques d'appropriation.

Mais on mesure aussi les limites de cette entreprise. Il faudrait mettre à l'épreuve une telle approche en considérant la question globale de l'exclusion, ce qui reviendrait à prendre en compte non seulement sa dimension relationnelle (fragilisation des liens, des cadres de référence) mais spatiale (marquage/neutralisation des territoires, relégation/accessibilité). De même, la question de la population - de plus en plus « jeune »- des différents types de «sans-abri » et des rapports qu'ils entretiennent avec des espaces-ressources mériterait d'être approfondie. À l'inverse, on en est resté pour l'essentiel, et de façon sans doute trop implicite quant à leur position sociale, au cas des jeunes issus de milieux populaires. Or, le renouvellement des approches socio-spatiales constaté est manifeste dans d'autres domaines et permet d'élargir la perspective. Ainsi dans le domaine de la sociologie de l'éducation, qu'il s'agisse d'aborder de façon générale le rapport entre ségrégation spatiale et ségrégation scolaire (Henriot-Van Zanten, 1990 ; Henriot-Van Zanten, Payet, Roulleau-Berger, 1994), ou d'aborder plus spécifiquement le rôle de la variable territoriale dans la construction de l'identité étudiante (Le Galès, Oberti, 1994 ; Felonneau, 1994). Il y a sans doute là matière à compenser la relative faiblesse des travaux sur les jeunes des classes moyennes et supérieures, et notamment dans le cas de ceux habitant les villes nouvelles, qui permettraient d'étendre l'analyse en termes d'expériences urbaines.

Reste la question essentielle des échelles d'analyse, d'intervention et d'appartenances collectives. Sur un plan sociologique, on peut se demander si l'enjeu d'une approche sociospatiale n'est pas de nuancer la portée de généralisations s'appuyant sur des enquêtes nationales pour mesurer le poids du local sans céder pour autant au localisme. Sur un plan politique, 
il serait de rendre manifeste ce qui a pu être - en France plus qu'en Angleterre, on l'a vu un « impensé » lourd de conséquences dans la mise en place de programmes urbains ou de dispositifs sociaux.

Michel KOKOREFF

LASTREE-IFRESI

Université de Lille I

2 , rue des Canonniers 59800 Lille, France

\section{RÉSUMÉ}

Dans un contexte marqué par la crise urbaine, la fin du plein emploi et la remise en cause des institutions de socialisation, on assiste depuis quelques années en France à un regain d'intérêt pour la question du rapport entre les jeunes et la ville. Proposer un bilan provisoire de ces travaux, en repérer les lignes de force et de transformation, mais aussi les obstacles et limites, constitue l'objet du présent article. On fait l'hypothèse que ces travaux attestent des formes différenciées de l'inscription sociale des jeunes dans les espaces urbains.

\section{SUMMARY}

In a context marked by urban crisis, the end of full-time employment and the challenging of institutions responsible for socialization, a renewal of interest over the last few years in France for the question of the relationship between the young and the city has developed. The object of this paper is to propose a preliminary assessment of these studies and to identify the main themes and transformations they present, as well as their limits and the obstacles they have encountered. An hypothesis can be formulated that these studies attest to a differentiation in forms of social integration of the young into urban spaces.

\section{RESUMEN}

Durante los últimos años hemos sido testigos de la multiplicación de trabajos sobre las relaciones entre los jóvenes y la ciudad. Este artículo se propone sugerir algunos elementos a fin de analizar la constitución progresiva de este tema de investigación hacia fin de los años 80 , así como subrayar las diferentes maneras de abordar la dimensión espacial de " la » juventud y la renovación de las perspectivas que éstas manifiestan. Se trata de poner en evidencia que, lejos de restringirse al análisis de los «problemas sociales », esos trabajos dan muestra de las formas diferenciadas de inscripción social de los jóvenes en el espacio urbano.

\section{BIBLIOGRAPHIE}

ALTHABE, G., B. LEGE et M. SELIM (1984), Urbanisme et réhabilitation symbolique, Paris, Anthropos.

AMIOT, M. (1986), Contre l'Etat, les sociologues - Éléments pour une histoire de la sociologie urbaine en France (19001980), Paris, Éditions de l'EHESS.

ANSELME, M., M. PERALDI (1985), Le petit séminaire à Marseille, Les Annales de la recherche urbaine, (1985), Jeunes urbains ès qualités, $\mathrm{n}^{\prime \prime} 27$, septembre.

AUGUSTIN, J.-P. (1991), Les jeunes dans la ville, Bordeaux, Presses Universitaires de Bordeaux.

BACHMANN, C., L. BASIER (1989), Mise en scène de la banlieue ordinaire - Stigmatisations urbaines et stratégies de communication, Paris, Syros.

BACHMANN, C., A. COPPEL (1989), Le Dragon domestique, Albin Michel.

BACHMANN, C. (1993), Jeunes et banlieues in G. FERREOL (éd.), Intégration et exclusion dans la société française contemporaine, pp. 129-154, Lille, Presses Universitaires de Lille

BAILLEAU, F, G. GARIOUD (1990), L'insécurité, une commune et l'État, in Sociétés contemporaines, $\mathrm{n}^{\circ}$ 4. pp. 141154

BARREYRE, J.-Y., A. VULBEAU (éds), (1995), La jeunesse et la nue, Paris Épi Desclée de Brouwer.

BATTEGAY A. (1985), "La génération de l'espace public » in Les Annales de la recherche urbaine, $\mathrm{n}^{\circ} 27$, septembre.

BATTEGAY, A., A. BOUBEKER (1992), Des Minguettes à Vaulx-en-Velin - Fractures sociales et discours publics in Les Temps Modernes, $\mathrm{n}^{\prime \prime} 545-546$, décembre/janvier.

BATTEGAY, A., A. BOUBEKER (1994), Les images publiques de l'immigration, Paris, L'Harmattan.

BEGAG, A. (1991), La ville et les autres - La famille immigrée et l'espace urbain, Lyon, PUL.

BEGAG, A. (1991), La révolte des lascars contre l'oubli à Vaulx-en-Velin, in Les Annales de la recherche urbaine, $\mathrm{n}^{\circ} 49$. BEGAG, A., C. DELORME (1994), Quartiers sensibles, Paris, Point-Virgule.

BLOCH, H., A. NIEDERHOFFER (trad. franç. 1963), Les bandes d'adolescents, Payot.

BLOSS, T. (1989), Jeunes maghrébins des quartiers nord de Marseille, Les Annales de la Recherche urbaine, $\mathrm{n}^{\circ} 41$, mars/avril. 
BODY-GENDROT, S. (1993), Ville et violence - L'imution de nouveaux acteurs, PUF.

BOUAMLANA, S. (1993), De la galère à la citoyenneté - Les jeunes, la cité, la société, Paris, Épi Desclée de Brouwer.

BOUHNIK, P., M. JOUBERT (1992), Économie des pratiques toxicomaniaques et lien social, in Dépendances, $\mathrm{n}^{\prime \prime} 3$, vol IV, pp. 25-36.

BOURDIEU, P. (éd.), (1993), La misère du monde, Paris, Le Seuil.

BRAKE, M. (1980), The sociology of youth and youth subcultures, Londres, Routeledge and Keegan Paul.

CALOGIROU, C. (1989), Sauver son honneur - Rapports sociaux en milieu urbain défavorisé, Paris, L'Harmattan.

CAUQUELIN, A. (1995), La ville la nuit, Paris, PUF.

CÉSARI, J. (1994), Marseille et ses communautés, in Esprit, mai.

CLAVE]_, M. (1992), L'ethnologie urbaine en France, des sciences exotiques à l'espace urbain, in L'homme et la société, $\mathrm{n}^{\circ} 104$.

CLOWARD, R.A., L.E. OHLIN (1960), Delinquency and opportunity, New York, The Free press.

COHEN ,P. (1980), «Subcultural conflict and working class community (1972) in BUTTERWORTH, E, WEIR, D, (dds) The new sociology of modem Britain, Londres, Fontana.

COING, H. (1966), Rénovation urbaine et changement social, Paris, Ed Ouvrières.

Conseil National des Villes (1994), L'économie souteraine de la drogue, DIV/MSH.

DE GAULEJAC, V., G. MURY (1977), Les jeunes de la rue, Paris, Privat.

DONZELOT, J. (éd.), (1991), Face à l'exclusion - Le modèle français, Paris, Éd Esprit.

DONZELOT, J. (éd.), (1991), avec P. ESTEBE, L'Etat-animateur - Essai sur la politique de la ville, Paris, Éd Esprit. DUBET, F., A. JAZOULI et D. LAPEYRONNIE (1985), L'État et les jeunes, Éditions ouvrières.

DUBET et al. (1986), Les opérations «été jeunes - Analyse longitudinale d'une politique publique, PUF-IDEF, 1986.

DUBET, F. (1987), La galère : jeunes en survie, Paris, Fayard.

DUBET, F., D. LAPEYRONNIE(1992), Les Quartiers d'exil, Paris, Le Seuil.

DUPREZ, D. (1989), Politiques locales de la jeunesse et gestion de l'ordre dans la cité, in Cahiers « Jeunesses et sociétés" n $\mathrm{n}^{\circ} 12$, décembre, pp. 33-52.

DUPRE'Z, D. M. HEDDLI (1992), Le mal des banlieues? Sentiment d'insécurité et crise identitaire, Paris, L'Harmattan. DUVIGNAUD, G. (1975), La planète des jeunes, Payot.

FELONNEAU, M.-L. (1994), Les étudiants et leurs territoires, in Revue Française de sociologie, XXXV, pp. 533-559.

FERRAND, A. (1974), La ségrégation comme assignation à des pratiques différentes in Sociologie du Sud-Est - 2-3, pp. $143-150$

FERRAND, A. (1976), La pratique spatiale des groupes de jeunes - Ségrégation et appropriation symbolique in $A c$. tes de la $3^{c}$ conférence internationale de psychologie de l'espace construit, Strasbourg, pp. 437-442.

FIZE, M., M. TOUCHE (1992), Le skate : la fureur de faire, Caen, Arcane-Beaunieux.

FIZE, M. (1993), Les bandes - L'« entre soi » adolescent, Paris, Desclée de Brower.

FORÊT, C., J.-P. PAYET (1986), Les territoires de la mobilité, Ministère de l'équipement

GALLAND, O. (1991), Sociologie de la jeunesse - L'entrée dans la vie, Paris, Armand Collin.

GRAFMEYER, Y., I. JOSEPH (1979), L'école de Chicago - Naissance de l'écologie urbaine, Champ urbain (rééd. 1990, Aubier).

GRAFMEYER, Y. (1994), Sociologie urbaine, Paris, Nathan Université.

GRAFMEYER, Y. (1994), Regards sociologiques sur la ségrégation in BRUN, J., RHEIN, C. (éds), La ségrégation dans la ville, Paris, L'Harmattan, pp. 85-117.

GUIDICELLI, A. (1991), La Caillera, Paris, Éd J. Bertoin.

HENRIOT-VAN ZANTEN A. (1990), L'école dans l'espace local, Lyon, Presse Universitaire de Lyon,.

HENRIOT-VAN ZANTEN A. (1994), avec PAYET J-P, ROULLEAU-BERGER L, (1994) L'école dans la ville, L'Harmattan.

HOGGART, R. (1970), La culture des pauvres, Paris, Éditions du Minuit.

JAZOULI, A., (1992), Les années banlieues, Paris, Le Seuil.

JOSEPH. I. (1984), Le passant considérable - Essai sur la dispersion de l'espace public, Paris Librairie Méridiens.

JOSEPH, I. (1994), L'espace public comme lieu de l'action, Les Annales de la recherche urbaine, n" 57-58, pp. 211-217. KEPEL, G. (1987), Les banlieues de l'islam, Paris, Point-Seuil (réédition 1991).

KOKOREFF, M. (1991), Tags et zoulous, une nouvelle violence urbaine, in Esprit, février, pp. 23-37.

KOKOREFF, M. (1993), L'espace des jeunes : territoires, identité et mobilité, in Les Annales de la recherche urbaine, $\mathrm{n}^{\circ} 60, \mathrm{pp} .171-179$.

KOKOREFF, M. (1994), La dimension spatiale des modes de vie des jeunes, in Sociétés contemporaines, nº 17, pp. 29 49.

LAÉ, J.-F. (1991), “Crise des banlieues : le béton n'est pas la cause », in Regards sur l'actualité, $n^{\circ} 172$, juillet, pp. $23-$ 34.

LAGRÉE, J.-C., P. LEW FAI (éds), (1982), La jeunesse en questions, Paris, La Documentation Française.

LAGRÉE, J.-C., P. LEW FAI (éds), (1985), La galère - Marginalisations juvéviles et collectivités locales, Paris, Éd du CNRS.

LAPEYF.ONNIE, D. (1987), Assimilation, mobilisation et action collective chez les jeunes des secondes générations de l'immigration maghrébine, Revue Française de sociologie, XXVIII, n*2, avril-juin.

LAPEYR:ONNIE, D. (1993), L'individu et les minorités - La France et l'Angleterre face à leurs immigrés, PUF.

LASSAVE, P. (1994), Le ministère urbain des sociologues - Approche d'une configuration intellectuelle dans la France des années 1980, EHESS.

LE GALIES, P., M. OBERTI, Lieux et pratiques sociales des étudiants dans la ville in Les Annales de la recherche urbaine, $\mathrm{n}^{\circ} 62-63$, pp. 251-264. 
LEONETTI-TABOADA, I. (1989), Cohabitation pluriethnique dans la ville : stratégies d'insertion locale et phénomènes identitaires, in Revue Européenne des migrations internationales, V 5, $\mathrm{n}^{\circ} 2$.

LESOURD, S. (éd.), (1992), Adolescents dans la cité, Paris, Éd Erès.

LINHART, V. (1992), Des Minguettes à Vaulx-en-Velin - Les réponses des pouvoirs publics aux violences urbaines, in Cultures et conflits, $\mathrm{n}^{\prime \prime} 6$. pp. 91-111.

MAUGER, G., FOSSE-POLIAK (1977), La vie buisonnière - Marginalité petite-bourgeoise et marginalité populaire, Paris, Maspéro.

MAUGER, G. (1990), Enquêter en milieu populaire, in Genèses n" 6, pp. 125-143.

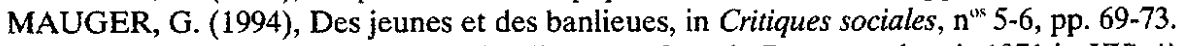

MIGNON, P. (1990), Supporters et hooligans en Grande-Bretagne depuis 1871 in $X X^{\mathrm{e}}$ siècle, avril-juin, pp. 37-47.

MIGNON, P. (1991), Note sur la criminalité juvénile et ses approches en Grande-Bretagne, in Les Cahiers de la sécurité intérieure, $\mathrm{n}^{\prime \prime} 5$, mai-juillet 1991, pp. 95-100.

MONOD, J. (1968), Les barjots, Paris, Julliard, 1968.

OSTROWETSKY.S. (1994), «L'urbain comme acte de langage - Sociologie et espace », in Les Annales de la recherche Urbaine, n" 64 , pp. 40-45.

PÉTONNET, C. (1979), On est tous dans le brouillard - Ethnologie des banlieues, Galilée.

PÉTONNET, C. (1982), Espaces habités - Ethnologie des banlieues, Galilée.

REMY, J., L. VOYE (1992), La ville : vers une nouvelle définition ? Paris , L'Harmattan.

ROMAN, J. (éd). (1994), Ville exclusion et citoyenneté, Paris, Éditions Esprit.

ROULLEAU-BERGER, L. (1991), La ville intervalle - Jeunes entre centre et banlieue, Paris, Méridiens Klincksieck.

ROULLEAU-BERGER, L. (1994), Sociologie urbaine et sociologie du travail en face à face, in Les Annales de la recherches urbaines, $\mathrm{n}^{\circ} 64$, pp. 109-115.

ROY, O. (1993), Les immigrés dans la ville, in Esprit, mai, pp. 41-53.

SANCHEZ-JANKOWSKI, M. (1991), Islands in the Street: Gangs in Urban American Society, University Press.

SANCHEZ-JANKOWSKI, M. (1994), "Les gangs et la presse ", in Actes de la recherche en sciences sociales, $n^{\circ} 101$, pp. 101-118.

SHAW, C.-R., M.-D. MAC KAY (1940), Juvenile delinquency in Urban Areas, Chicago, University of Chicago Press.

TARRIUS, A. (1993), Territoires circulatoires et espaces urbains, in Les Annales de la recherche urbaine, $\mathrm{n}^{\circ}$ 59-60, pp. 5160.

TOURAINE, A. (1992), Face à l'ecxlusion, in J. ROMAN (éd.), Citoyenneté et urbanité, Paris, Éd Esprit.

THRASHER, F.-M. (1927), The gang, Chicago, University of Chicago.

TRIBALAT, M. (1995), Faire France, Paris, Maspero.

VIEILLARD BARON, H. (1990), Le ghetto, Lieu commun, impropre et banal, in Les Annales de la recherche urbaine, $n^{\prime \prime} 49$, pp. 13-22.

VIEILLARD BARON, H. Les banlieues Françaises ou le ghetto impossible, Éd de l'Aube, 1994.

VULBEAU, A. (1992), Du tag au tag, Paris, Épi Desclée de Brouwer.

VULBEAU, A. (1994) Concevoir et esxpérimenter un espace public de la jeunesse, Communication au séminaire Jeunesses et politique d'insertion, Commissariat Général du Plan.

WACOUANT, L. (1992), Pour en finir avec le mythe des cités-ghettos, in Les Annales de la recherche urbaine, $\mathrm{n}^{\circ} 54$, pp. 21-30.

WACQUANT, L. (1994), «Le gang comme prédateur collectif » in Actes de la recherche en sciences sociales, $\mathrm{n}^{\circ} 101-$ 102, pp. 87-100.

WHYTE, W.F., Street Comer Society, Chicago, University of Chicago Press, 1943. 